\title{
Descripción y proyección de la esperanza de vida al nacimiento en México (1900-2050)
}

\section{Óscar Peláez Herreros*}

En este texto se sostiene que la evolución de la esperanza de vida al nacimiento en México está condicionada por el margen de mejora existente respecto a los países más avanzados en la lucha contra la mortalidad, de ahi que para proyectar la evolución futura de la serie nacional se ajuste un modelo logístico de dos asintotas a la esperanza de vida máxima internacional, que luego se adapta al caso mexicano atendiendo a las características particulares que se detectan.

Palabras clave: esperanza de vida, proyección, modelo logístico, transición epidemiológica, mortalidad infantil.

Siguiendo una argumentación semejante a la expuesta por Ortes (1790), Thomas R. Malthus explicaba en su texto de 1798 que la población se encontraba sumida en una trampa demográfica que impedía el progreso que perseguían algunos reformadores sociales, como Godwin o Condorcet. Para Malthus [1798] el producto per cápita era invariable. Cada expansión de los medios de subsistencia era absorbida por el aumento de la población: "en todas las sociedades [...] se observa una presión constante hacia el aumento de la población [que] tiende, con no menos constancia, a hundir a las clases inferiores de la sociedad en la miseria y a evitar toda permanente mejora considerable de su situación" (Malthus, 1970: 62).

El razonamiento de Malthus explica de forma adecuada lo acontecido hasta entonces: una ausencia de progreso. Pero no se ajusta a lo ocurrido en los años inmediatamente posteriores, cuando las revoluciones agraria e industrial iniciaron el desarrollo económico y la transición demográfica en Inglaterra, y la población y la prosperidad aumentaron de forma simultánea y sostenida. Los progresos en la agricultura y en los transportes, que facilitaron una alimentación más variada y abundante, los cambios en la organización política y social y las mejoras en la higiene, liberaron a la población de los episodios de mortalidad catastrófica característicos del régimen demográfico anti-

* Facultad de Ciencias Sociales, Universidad Autónoma de Chiapas. Correo electrónico: opelaez1@gmail.com.

ESTUDIOS DEMOGRÁFICOS Y URBANOS, VOL. 24, NÚM. 2 (71), 2009, 469-492 
guo. La tasa de mortalidad comenzó a descender (Notestein, 1945: 39). En un principio la tasa de natalidad aumentó, para luego mantenerse estable en torno a $40 \%$ o durante la primera mitad del siglo XIX, y finalmente emprendió el largo descenso que la llevó a 10\%o (Vallin, 1995: 69; Galor y Weil, 1999 y 2000). Con la industrialización surgieron la sociedad de clases y la posibilidad de ascenso social (Kerbo, 2003: 55-57). Aparecieron "frenos preventivos" que evitaron que la población se reprodujera hasta retornar al límite de la subsistencia, lo que permitió consolidar los avances en las condiciones de vida y escapar de la trampa maltusiana.

Los cambios que se iniciaron en Inglaterra pronto se extendieron a otros países, aunque con calendarios e intensidades diferentes; primero fue dentro del continente europeo y luego fuera de él. En México, por ejemplo, el aumento de la capacidad de supervivencia no comenzó hasta la conclusión del siglo XIX (Morelos, 1994: 80). En 1930 la esperanza de vida al nacimiento era de 33 años para los hombres y de 34.7 para las mujeres (INEGI, 2007: 87), cifras ligeramente superiores a las del régimen demográfico antiguo y muy alejadas de los valores que ya por entonces presentaban algunas poblaciones, como las de Australia o los Países Bajos, donde la esperanza de vida masculina superaba los 63 años de edad y la femenina los 65, duplicando prácticamente los valores observados entre la población mexicana. ${ }^{1}$ ¿Qué factores explican que en las últimas décadas la esperanza de vida al nacimiento en México haya aumentado a un ritmo acelerado hasta alcanzar los 74.5 años en 2006 (INEGI, 2007: 88) y situarse a apenas 8 de la cifra correspondiente a la población japonesa, la más longeva del mundo? Y, tal vez, lo que es más relevante para la población actual, ¿qué cabe esperar en el futuro?

En este artículo se trata de dar respuesta a ambas cuestiones. Para ello, en primer lugar, se revisa la evolución pasada de la esperanza de vida al nacimiento de la población residente en México, prestando especial atención a las causas que han permitido que la longevidad media se haya duplicado en los últimos setenta años. Conociendo esa evolución y sus causas, se presenta una metodología que permite describir y proyectar la tendencia a largo plazo de la esperanza de vida al nacimiento de forma consecuente con su dinámica histórica, no sólo en el país, sino en el mundo entero. En el tercer apartado se exponen

${ }^{1}$ Los datos de esperanza de vida que se mencionan a lo largo del presente artículo para los distintos países, a excepción de México, fueron extraídos de la Human Mortality Database el 29 de agosto de 2008 (HMD, 2008). 
los resultados de las proyecciones realizadas, se comparan con los de otros estudios que se han publicado recientemente y se analizan las peculiaridades de la mortalidad mexicana. Un apartado de conclusiones cierra el artículo.

\section{Las causas de la expansión de la esperanza de vida en México}

Tanto Morelos (1994: 80) como Arriaga (1968) sostienen que los primeros avances en la prolongación de la supervivencia tuvieron lugar durante el porfiriato. Las tímidas mejoras que se consiguieron en aquellos años se diluyeron en la década revolucionaria, pero sirvieron para marcar el camino que se seguiría inmediatamente después. Hasta ese momento las fluctuaciones debidas a las malas cosechas o a las epidemias (Oliver, 1988) habían caracterizado la dinámica de una expectativa de vida muy baja, en muchas ocasiones inferior a los 25 años de edad, y estancada a largo plazo. El final de la Revolución significó la ruptura definitiva con el régimen demográfico antiguo.

Durante la década de los veinte la esperanza de vida al nacimiento ya rebasaba los 30 años de edad. En el siguiente decenio el crecimiento comenzó a acelerarse debido a la aplicación de los primeros programas de salud pública y al incremento de la alfabetización, y así alcanzó un ritmo de mejora de 0.5 años por cada año transcurrido (Camposortega, 1992: 377). En las décadas siguientes, al crecimiento socioeconómico que había favorecido la reducción de la mortalidad en los primeros años, se sumó la aplicación de las nuevas tecnologías médicas (Arriaga y Davis, 1969), lo que aceleró aún más el ritmo de avance.

Camposortega (1992: 384) califica el periodo que transcurrió entre 1940 y 1960 como de "disminución extraordinaria" de la mortalidad. Entre esas dos fechas la esperanza de vida de la población residente en México se incrementó a un ritmo de casi un año por cada año de calendario. En el caso femenino se elevó desde los 41 años de 1940 hasta casi los $60 .^{2}$ En el masculino pasó de los 39 a los

${ }^{2}$ Según las estimaciones de Benítez y Cabrera (1967) la esperanza de vida femenina se elevó desde 42.50 años de 1940 hasta 60.32 de 1960. Para Arriaga (1968) el aumento se produjo desde 39.84 años hasta 59.58. Para Corona et al. (1982) desde 41.46 hasta 60.14. Según las estimaciones corregidas de Camposortega (1992) la capacidad media de supervivencia habría pasado de 41.65 años a 59.73, mientras que para INEGI (2007) el aumento sería desde 39.80 años hasta 59.40, y para Partida (2008) de 41.75 a 59.33 una vez ajustadas las esperanzas de vida. 
56.5 años. $^{3}$ La mayor disponibilidad de alimentos tras la reforma agraria, la construcción de carreteras y sistemas de riego, la creación del Instituto Mexicano del Seguro Social, la puesta en funcionamiento de la Secretaría de Salubridad y Asistencia y de diversas campañas de vacunación y de utilización de insecticidas, contribuyeron de manera notable a reducir la mortalidad por causas infecciosas y parasitarias, que suele afectar a los jóvenes (Camposortega, 1992: 384385). El descenso de la mortalidad en el extremo de la distribución impulsó el rápido avance de la esperanza de vida que tuvo lugar en esas dos décadas.

En los sesenta y en los setenta el crecimiento de la esperanza de vida redujo su ritmo. Para 1980 la expectativa de supervivencia de la población femenina apenas aumentó 10 años, y la masculina 8 , a un ritmo que resultó ser la mitad de intenso que en las dos décadas precedentes. El creciente peso de algunas causas de fallecimiento, como la violencia, los accidentes y las enfermedades degenerativas (Morelos, 1994: 58), más difíciles de combatir, unido al hecho de que el simple aumento de la esperanza de vida hace que esta variable sea menos sensitiva a nuevos cambios en las tasas de mortalidad por edades (Olshansky et al., 1990 y 2001), explican el progreso cada vez menor que se ha detectado.

En las siguientes dos décadas, hasta alcanzar el nuevo milenio, el crecimiento de la esperanza de vida se desaceleró aún más. En el año 2000 la femenina alcanzó 76.5 años y la masculina 71.3, situándose 6.5 y 7.3 años, respectivamente, por encima de los valores de 1980. Mientras que la esperanza de vida masculina apenas varió su ritmo de crecimiento respecto a las décadas inmediatamente anteriores, la femenina casi redujo su empuje a la mitad, lo que llevó a que por primera vez se estrechara la brecha diferencial entre las longevidades de ambos sexos, como se ha comentado, más por el pobre desempeño de la serie femenina que por el progreso de la masculina, ligeramente peor que en décadas precedentes.

El aumento más rápido de la esperanza de vida entre las mujeres que entre los hombres a lo largo de todo el siglo Xx, con la excepción de las dos últimas décadas, no fue un fenómeno exclusivo de México.

${ }^{3}$ Benítez y Cabrera (1967) advierten un aumento de la esperanza de vida masculina desde 40.39 años en 1940 hasta 57.61 en 1960. Arriaga (1968) de 37.67 años a 56.37. Corona et al. (1982) desde 39.46 hasta 57.08. Para Camposortega (1992) la esperanza de vida, una vez corregida, habría aumentado de 39.12 años a 55.99. Para INEGI (2007), de 37.70 a 55.60. Y según Partida (2008), de 39.53 a 56.10 años. 
Otras poblaciones, como las de Inglaterra, Estados Unidos, Suecia, Francia o Italia, por ejemplo, experimentaron ese mismo proceso con un calendario semejante (Meslé, 2004; Gerland, 2005). Esto da idea de que la evolución de la esperanza de vida en un país guarda una estrecha relación con lo que ocurre en el resto del mundo.

Cuando México abandonó el régimen demográfico antiguo, la ventaja de las poblaciones que habían iniciado la transición demográfica un siglo antes resultaba enorme. Al inicio de la década de los treinta la esperanza de vida al nacimiento en Noruega era de 64 años, y alcanzaba los 65 en Australia y los Países Bajos; en México sólo llegaba a los 37 años (Partida, 2008: 64). Esa diferencia, acumulada a lo largo de un siglo de retraso, se recortó de manera "extraordinaria" gracias al desarrollo de unas instituciones que ya se habían mostrado exitosas en otros países, y que favorecieron la puesta en marcha de programas de salud, de alfabetización, o de remoción de las condiciones que impedían la adecuada nutrición de la población, ya fuese mediante la reforma agraria o la construcción de infraestructuras viarias y de regadío (Camposortega, 1992: 384). Todo el progreso acumulado durante décadas por las poblaciones líderes en longevidad estaba al alcance de los mexicanos. Los insecticidas, las vacunas, los medicamentos que podían reducir la incidencia de las enfermedades de origen infeccioso y que habían requerido un largo proceso de investigación y desarrollo en los países pioneros, estaban disponibles sin mucho esfuerzo. No obstante, había que realizar ese esfuerzo, y México lo hizo en cuanto dispuso de las instituciones adecuadas para incorporar y difundir entre la población los avances que desde hacía tiempo ya disfrutaban las naciones líderes.

El espectacular progreso de la esperanza de vida en México durante las décadas de los cuarenta y de los cincuenta fue debido al no menos espectacular retraso acumulado y a la continuidad que se dio en la aplicación de una serie de políticas coherentes. La ralentización advertida en las décadas siguientes responde al progresivo agotamiento del margen de mejora. En 1930 la distancia respecto al país líder del mundo en esperanza de vida era de 29 años. La población de Nueva Zelanda casi duplicaba en longevidad a la mexicana. En 1940 la diferencia ya se había reducido a 27 años. Dos décadas más tarde, tras el intenso crecimiento de la esperanza de vida nacional a un ritmo de casi un año por cada año de calendario, la distancia respecto al nuevo líder, Islandia, se había recortado a 16 años. En fechas posteriores, pese a la fuerte ralentización advertida en el ritmo de mejora de esta varia- 
ble, la distancia respecto a las naciones más avanzadas continuó reduciéndose, aunque, eso sí, a un ritmo más lento, ya que el margen de mejora, y en consecuencia las innovaciones que se podían incorporar, eran cada vez menores.

En 1970, los 60.6 años de esperanza de vida registrados en México ya sólo distaban 14 años de los 74.7 de Suecia. Un decenio más tarde esa diferencia se había reducido a 9.5 años. En 1990 a 8.4. Y en 1995 a 7.4, lo más cerca que la población mexicana ha estado del máximo mundial desde el inicio de la transición demográfica. A partir de ese momento, en vez de proseguir la convergencia secular que se ha descrito, y que Wilson (2001) advierte para la mayor parte de las poblaciones, se inicia una fase de leve divergencia respecto a los países más avanzados. La esperanza de vida en México sólo se expande 2.2 años entre 1995 y 2005, mientras que en Japón, el líder, lo hace 2.5 años, de forma que la distancia se amplía sucesivamente hasta 7.5 años en 2000 y 7.7 en 2005 . Distinguiendo por sexos se puede comprobar que la esperanza de vida femenina es la que ocasiona el deterioro relativo en términos agregados durante el periodo 1995-2000, en el cual la serie masculina continúa reduciendo su diferencia respecto al máximo. En el lustro posterior ya son ambas series las que presentan una evolución divergente con relación al líder, concluyendo en 2005 a 8.45 y 7.25 años de las expectativas de supervivencia de Japón, en el caso femenino, y de Islandia, en el masculino, respectivamente.

El progresivo acercamiento al nivel máximo de esperanza de vida reduce el margen de mejora de la serie nacional y explica la ralentización de su ritmo de crecimiento. Pero, ¿qué explica que en los años más recientes la desaceleración haya sido tan importante como para incluso divergir de la serie de máximos? Una vez más la revisión de la experiencia internacional puede ayudar a comprender los motivos de esta fuerte desaceleración y divergencia.

A principios de la segunda mitad del siglo Xx la esperanza de vida de los países por entonces líderes no sólo desaceleró su crecimiento, sino que incluso acabó estancándose. Noruega, por ejemplo, tardó 17 años, desde 1953 hasta 1970, en pasar de 73 a 74 años de expectativa de vida. A los Países Bajos les llevó de 1958 a 1973 realizar ese mismo tránsito. Suecia tuvo que esperar desde 1958 hasta 1975 para alcanzar los 75 años desde los 73. En aquellas fechas la incidencia de las enfermedades infecciosas se había reducido, dejando paso a las degenerativas, de forma que la transición epidemiológica (Omran, 1971) parecía estar completa y el límite biológico de la especie huma- 
na, que Bourgeois-Pichat (1952) había cifrado en 76.3 años de esperanza de vida para los hombres y 78.2 para las mujeres, se mostraba insuperable.

No obstante, pocos años más tarde la esperanza de vida volvió a crecer. Los recursos liberados de la investigación y del tratamiento de las enfermedades infecciosas y parasitarias, ya desbancadas de las primeras causas de muerte, se dirigieron hacia las enfermedades crónicas y degenerativas y dieron sus frutos. La mortalidad entre las personas de mayor edad comenzó a reducirse, añadiendo una nueva etapa a la transición epidemiológica: "la edad de las enfermedades degenerativas diferidas" (Olshansky y Ault, 1986).

El paso de la tercera a la cuarta etapa no fue inmediato, requirió un periodo de adaptación. En los países líderes, que tuvieron que soportar el peso de la innovación, la esperanza de vida prácticamente quedó estancada durante década y media. En los países seguidores el crecimiento de la longevidad no se detuvo, pero sufrió una importante ralentización al tratar de rebasar los 70 años de edad. Superado el tránsito hacia la cuarta etapa, el ritmo de crecimiento de la esperanza de vida volvió a acelerarse en muchos casos, lo que encuentra confirmación en el trabajo de White (2002). ${ }^{4}$

En su momento, el estancamiento de los líderes fue aprovechado por México para recortar su desventaja. Entre 1950 y 1980 la esperanza de vida nacional aumentó 17.7 años, rebajando 12.6 años la diferencia respecto al máximo, ya que éste sólo aumentó 5 años durante esas tres décadas. Ahora que la expectativa de vida ha vuelto a crecer en las naciones más avanzadas y es México el que tiene que afrontar la transición hacia "la edad de las enfermedades degenerativas diferidas", la distancia tiende a ampliarse.

El descenso a largo plazo del ritmo de crecimiento de la esperanza de vida en México responde al hecho de que cada vez se dispone de un menor margen de mejora respecto a los países más avanzados. Unido a ello, la reciente divergencia respecto a los líderes se debe al paso desde el tercero al cuarto estadio de la transición epidemiológica, un proceso que tradicionalmente ha interrumpido el ritmo de expansión de la esperanza de vida, ya que la reasignación de recursos desde

${ }^{4}$ White (2002) analiza la evolución de la esperanza de vida desde 1955 hasta 1996. Concluye que para 19 de los 21 países industrializados objeto de estudio, la tendencia lineal explica mejor que cualquier otra la evolución de la esperanza de vida, y que en los ajustes cuadráticos el coeficiente del término cuadrático suele resultar positivo, lo que indica una aceleración progresiva, y no una desaceleración, en el crecimiento de la longevidad a lo largo del periodo analizado. 
el diagnóstico y el tratamiento de las enfermedades infecciosas y parasitarias hacia las crónicas y degenerativas requiere tiempo.

Atendiendo a la experiencia internacional, en los próximos años cabe esperar que México supere la fase de lento crecimiento en la que se encuentra reorientando sus políticas de salud, y que luego sea capaz de reanudar la convergencia con los países más avanzados, como ya han hecho otras poblaciones. ${ }^{5}$ A largo plazo, el crecimiento de la esperanza de vida nacional dependerá tanto de la capacidad propia para asimilar los avances que se den en el plano internacional, como de la cantidad de avances que se produzcan y del margen de mejora que generen. Por tanto, para estudiar la posible evolución futura de la esperanza de vida en México se hace necesario considerar no sólo la capacidad de mejora nacional, sino también la internacional. Por ello se propone la siguiente metodología.

\section{Metodología para la proyección de la esperanza de vida}

Como se ha visto, la evolución de la esperanza de vida de cualquier población depende de las medidas que se adopten en ella, pero también de lo que ocurra en el resto de las poblaciones, ya que existen transferencias de salud entre países que interrelacionan las dinámicas de las longevidades. La propuesta que realizan Oeppen y Vaupel (2002: 1031) consistente en "prever la evolución de la esperanza de vida considerando la brecha entre la serie nacional y la de mejores prácticas" tiene en cuenta los dos ámbitos. Para desarrollarla se construye la "serie de mejores prácticas" con las esperanzas de vida máximas de cada año observadas en el mundo, se estima un modelo que describa esa serie y se extrapola su dinámica hacia el futuro. Conocida la tendencia de los máximos, se atiende a las peculiaridades de la serie nacional objeto de análisis, se compara su evolución con la de la serie de máximos calculando la distancia existente entre ambas y, entonces, dependiendo de la relación observada y de las causas que la explican, se puede

\footnotetext{
${ }^{5}$ En este sentido parecen muy acertados el análisis y la propuesta de Partida (2008: 73), según los cuales "el magro avance en la reducción de la mortalidad en la senectud en años recientes deriva de la falta de orientación de los servicios de salud hacia las demandas específicas de los adultos mayores, que es difícil revertir esa tendencia en el mediano plazo, y es factible que, al menos en los próximos años, el riesgo de morir en la vejez se quede estancado. Así, la proyección de la mortalidad consistió en disminuir las tasas específicas por edad de menores de 65 años a lo largo de los 45 años de la proyección y posponer hasta 2015 el descenso de las tasas de los adultos mayores".
} 
suponer que en el futuro la serie nacional va a aproximarse más a los países líderes, que va a mantener la distancia, o que, incluso, va a alejarse de ellos. De esta manera se consideran las características de cada población dentro del marco global, esto es, teniendo en cuenta las posibles mejoras disponibles en el mundo. No obstante, el procedimiento original de Oeppen y Vaupel (2002) presenta algunas deficiencias que se pueden subsanar, especialmente en la construcción del modelo de mejores prácticas, donde se ajusta una tendencia lineal que difícilmente puede extrapolarse fuera del periodo utilizado para su estimación.

Con los datos de esperanza de vida de diversas poblaciones, Oeppen y Vaupel (2002) elaboran sendas series de máximos por sexo para el periodo 1840-2000. Esas series se modelizan mediante regresiones lineales, obteniendo unos coeficientes de determinación de $r^{2}=0.992$ para las mujeres y de $r^{2}=0.980$ para los hombres, lo que les lleva a concluir que "el incremento de la esperanza de vida en las últimas 16 décadas es tan extraordinariamente lineal que podría tratarse de la regularidad demográfica más destacable jamás observada" . ${ }^{6}$ El problema es que aunque la relación lineal resulta casi perfecta en el periodo descrito, no hay garantía alguna de que se mantenga fuera de él. De hecho, las pruebas existentes parecen indicar que el crecimiento sostenido de la esperanza de vida máxima sólo se ha producido durante el siglo y medio más reciente.

El modelo lineal no puede explicar la evolución de la serie en fechas previas a las consideradas, especialmente antes de 1650, cuando empieza a generar valores negativos para la esperanza de vida. Asimismo, y como los propios autores reconocen, tampoco es capaz de explicar el futuro a muy largo plazo, cuando la especie humana tendería a la inmortalidad. El modelo lineal subestima el pasado y, probablemente sobreestime el futuro de la esperanza de vida máxima. Debido a ello las diferencias calculadas para cualquier población pueden resultar erróneas fuera del intervalo descrito. Se hace necesario ajustar un nuevo modelo a la serie de máximos. Un modelo que tenga en cuenta que la esperanza de vida ha permanecido estable a largo plazo durante

${ }^{6}$ Desde los primeros estudios relacionados con la mortalidad se han encontrado regularidades difícilmente explicables, incluso en el marco de la ley de los grandes números. Así lo constata Spiegel (1973: 168) cuando afirma que "el trabajo de Graunt influyó y fue continuado por un cierto número de teólogos, que consideraron la regularidad de los fenómenos demográficos como una prueba de la existencia de un plan divino". 
la mayor parte de la historia de la humanidad, y que sólo ha comenzado a crecer en los últimos siglos, y no de manera exactamente lineal sino con "una leve forma de S" como advierte Lee (2003: 2). ${ }^{7}$

La función matemática que mejor se adapta a la evolución descrita es una curva logística de dos asíntotas del tipo:

$$
\hat{e}_{0}^{t}=\frac{e_{0}^{\max }-e_{0}^{\min }}{1+\exp \{d+c \cdot t\}}+e_{0}^{\min }
$$

semejante a la que presentan Bulatao et al. (1989), donde $\hat{e}_{0}^{t}$ es la esperanza de vida al nacimiento estimada para el año $t ; d$ y $c$ son constantes desconocidas; $e_{0}^{\min }$ es la esperanza de vida mínima; y $e_{0}^{\max }$ es el nivel de saturación, el máximo valor que puede alcanzar la variable dependiente.

A diferencia de la propuesta formulada por Bulatao et al. (1989), en este caso se pretende ajustar la curva logística a la serie de máximos mundiales, y no directamente a la serie nacional, para luego proceder como sugieren Oeppen y Vaupel (2002: 1031) o Vaupel en SCOR (2004: 4), esto es: proyectando la esperanza de vida máxima de la población femenina, calculando la esperanza de vida femenina nacional a partir de las diferencias con la serie de máximos proyectada y, finalmente, obteniendo la serie masculina nacional con relación a los valores estimados para la femenina. De este modo, se tienen en cuenta las características propias de cada población, pero dentro del marco internacional, considerando el margen de mejora que se dispondrá en cada momento respecto a los países líderes.

Asimismo, a diferencia de otros trabajos que emplean curvas logísticas para describir y proyectar la evolución de la esperanza de vida, en este caso no se fijan de antemano la esperanza de vida mínima, ni el nivel de saturación. Por ejemplo, Bulatao et al. (1989) fijan en 20 años el valor mínimo de la esperanza de vida y, alternativamente, en 82.5 y 90 años el máximo. Zamora (1994) elige 30 y 86 años como asíntotas para su modelo. En la presente investigación no se fija ninguna de las dos asíntotas. Se estiman distintos modelos para las posibles combinaciones de ambos valores y se elige de entre todos ellos el que presenta

${ }^{7}$ Lee (2003) encuentra que las series de máximos construidas por Oeppen y Vaupel (2002) pueden dividirse en tres tramos. El primero de ellos, desde 1840 hasta 1900, se caracteriza por el crecimiento de la esperanza de vida. El segundo, desde 1900 hasta 1950, por la aceleración de ese crecimiento; que acaba ralentizándose en las décadas más recientes (1950-2000). 
un error cuadrático medio menor. ${ }^{8}$ De esta forma, las esperanzas de vida máxima y mínima se determinan implícitamente al estimar el modelo, quedando libres de juicios de valor.

\section{Resultados}

Aplicando la metodología anterior a la serie de máximos femeninos desde 1840 hasta $2006^{9}$ se obtiene que la curva logística que comete un menor error es la que utiliza un nivel de saturación de 106 años y una esperanza de vida mínima de 29 (cuadro 1), con unos coeficientes $c=-0.0133$ y $d=-25.7516$ estadísticamente significativos y un error cuadrático medio igual a 2.087.

Este ajuste puede compararse con el resultante de aplicar sobre la misma serie de datos el análisis lineal propuesto por Oeppen y Vaupel (2002), obteniéndose entonces un término independiente $a=$ -398.1874 , una pendiente $b=0.2414$ y un error cuadrático medio de 2.194, superior al del modelo logístico que, por tanto, se ajusta mejor que el lineal al perfil levemente curvado de la serie de observaciones durante los años 1840-2006. A ello hay que unir el hecho de que el ajuste logístico también explique mejor que el lineal la evolución de la esperanza de vida durante el periodo anterior al utilizado para la estimación, ya que la esperanza de vida mínima, de 29 años de edad, obtenida sin establecer ningún supuesto previo sobre su valor, es una cifra compatible con la evidencia histórica de diversas poblaciones como muestran los estudios de Spengler (1978: 104), Bagnall (1993: 182) y Livi-Bacci (2002: 147), entre otros.

${ }^{8}$ Se utiliza el error cuadrático medio como medida de la bondad de los ajustes debido a que, al tratarse de modelos no lineales, la descomposición de la varianza no está garantizada y, por tanto, el coeficiente de determinación lineal, $r^{2}$, puede tomar valores no comprendidos entre 0 y 1 .

${ }^{9}$ Para la construcción de la serie de máximos femeninos se ha considerado la información de la Human Mortality Database correspondiente a las poblaciones de: Alemania Occidental (1956-2006), Alemania Oriental (1956-2006), Australia (1921-2004), Austria (1947-2005), Bélgica (1841-1913 y 1919-2005), Bielorrusia (1959-2005), Bulgaria (1947-2005), Canadá (1921-2005), Dinamarca (1835-2006), Eslovaquia (1950-2006), Eslovenia (1983-2006), España (1908-2006), Estados Unidos (1933-2005), Estonia (19592005), Finlandia (1878-2006), Francia (1899-2005), Hungría (1950-2005), Inglaterra y Gales (1841-2005), Islandia (1838-2006), Italia (1872-2004), Japón (1947-2006), Letonia (1959-2005), Lituania (1959-2005), Luxemburgo (1960-2005), Noruega (1846-2006), Nueva Zelanda (1876-2003), Países Bajos (1850-2006), Polonia (1958-2006), Portugal (1940-2006), República Checa (1950-2006), Rusia (1959-2006), Suecia (1751-2006), Suiza (1876-2006), Taiwan (1970-2005) y Ucrania (1959-2006). 
CUADRO 1

Errores cuadráticos medios del modelo logístico para distintas combinaciones de esperanzas de vida máximas y mínimas

\begin{tabular}{cccccccc}
\hline & \multicolumn{7}{c}{ Asintota inferior } \\
\cline { 2 - 8 } Nivel de saturación & 26 & 27 & 28 & 29 & 30 & 31 & 32 \\
\hline 100 & & & & & & & 2.1053 \\
101 & & & & & $\ldots$ & $\ldots$ & 2.0941 \\
102 & & & & & 2.1008 & 2.0920 & $\mathbf{2 . 0 8 9 8}$ \\
103 & & & $\ldots$ & $\ldots$ & 2.0913 & 2.0884 & 2.0903 \\
104 & & & 2.0995 & 2.0913 & $\mathbf{2 . 0 8 7 4}$ & $\mathbf{2 . 0 8 7 7}$ & 2.0961 \\
105 & & $\ldots$ & 2.0915 & 2.0877 & 2.0880 & 2.0922 & $\ldots$ \\
106 & $\ldots$ & 2.0927 & $\mathbf{2 . 0 8 7 6}$ & $\mathbf{2 . 0 8 7 3}$ & 2.0915 & 2.1024 & \\
107 & 2.0942 & 2.0887 & 2.0878 & 2.0896 & $\ldots$ & $\ldots$ & \\
108 & 2.0902 & $\mathbf{2 . 0 8 8 3}$ & 2.0909 & 2.0958 & & & \\
109 & $\mathbf{2 . 0 8 9 2}$ & 2.0905 & $\ldots$ & $\ldots$ & & & \\
110 & 2.0908 & 2.0954 & & & & & \\
111 & 2.0958 & $\ldots$ & & & & & \\
\hline
\end{tabular}

FUENTE: Elaboración propia.

El nivel de saturación del modelo, igual a 106 años, se sitúa bastante por encima de las cifras que suelen ofrecer otros estudios como límites máximos para la expansión de la esperanza de vida. Sin embargo no hay que olvidar que se trata de un límite a muy largo plazo y que aún existen posibilidades de prolongar la longevidad, bien adoptando costumbres más saludables o por medio de los avances en la medicina. Por otro lado, en comparación con las estimaciones realizadas por Oeppen y Vaupel (2002), que aseguran que la esperanza de vida femenina de alguna población alcanzará los 100 años en torno a 2060, el modelo logístico propuesto retrasa ese momento medio siglo, concretamente hasta el año 2115.

Suponiendo que la serie de esperanza de vida de los países más avanzados vaya a seguir evolucionando como lo ha venido haciendo a lo largo de toda la historia, para conocer el margen de mejora del que dispondrá la población mexicana en las próximas décadas basta con extrapolar hacia el futuro la trayectoria logística que se ha estimado y que describe en forma adecuada la evolución pasada de la serie de máximos. La gráfica 1 muestra la serie de esperanza de vida máxima para la población femenina, la tendencia logística que mejor se ajusta 


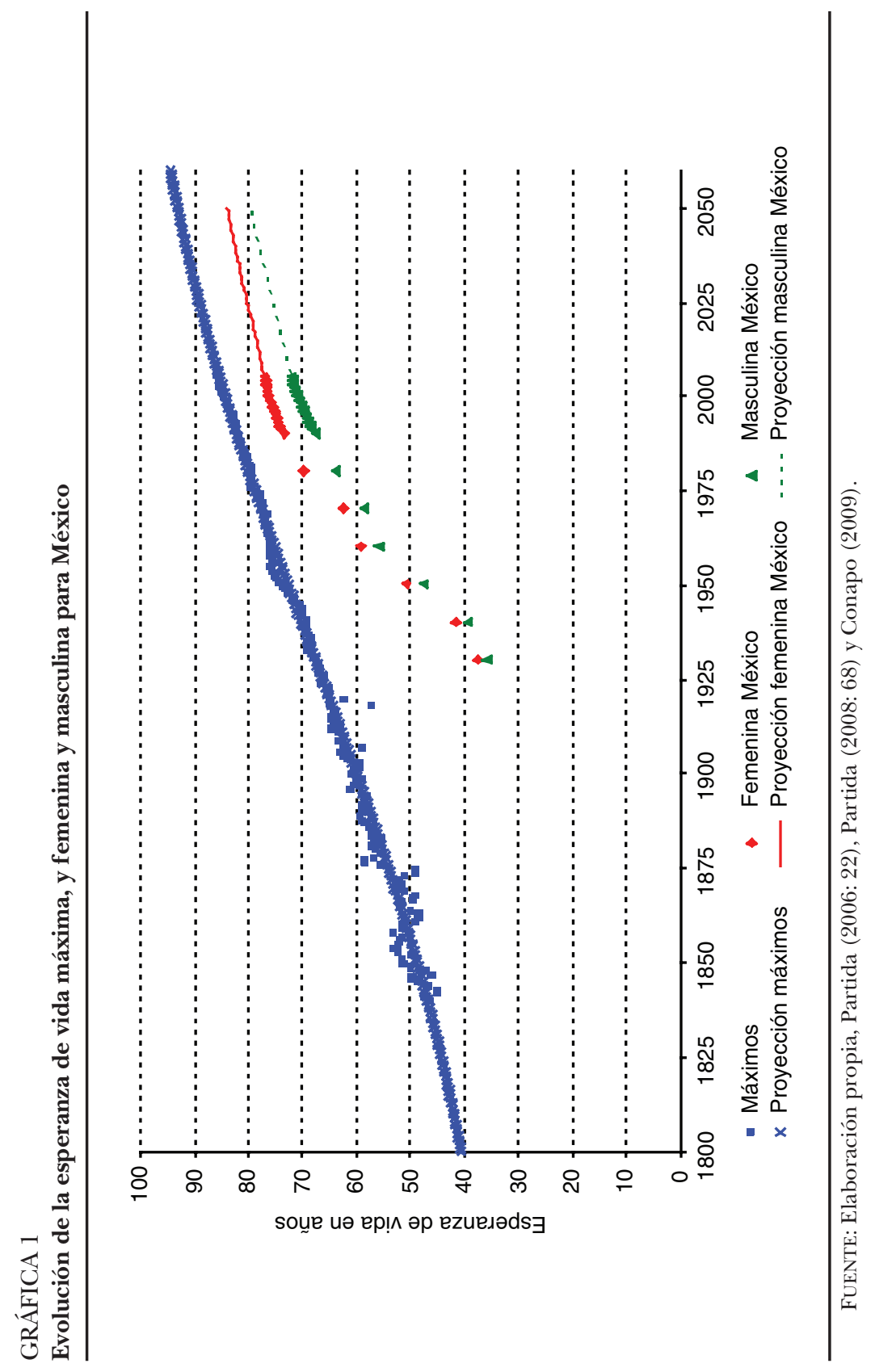


a esta serie, los datos observados en la población mexicana para cada sexo y las respectivas proyecciones elaboradas por el Consejo Nacional de Población (Conapo).

Como se puede apreciar, si la esperanza de vida máxima sigue creciendo según el modelo descrito, el margen de mejora para la población mexicana se ampliará considerablemente en los próximos años. Superado el tránsito de "la edad de las enfermedades degenerativas y causadas por el hombre" a la "de las enfermedades degenerativas diferidas", la esperanza de vida nacional podría volver a aumentar a un ritmo elevado, retomando la senda de la convergencia con la serie de máximos. De ocurrir esto, las proyecciones realizadas por el Conapo, de 79.86 y 83.95 años para las poblaciones masculina y femenina en 2050, resultarían bastante modestas. ${ }^{10}$ De hecho, sólo con que se consiguiera mantener la actual distancia respecto a la serie de máximos, las esperanzas de vida deberían elevarse a 79.97 y 84.81 años respectivamente. Si se reanudara el proceso de convergencia, interrumpido por el escaso retroceso de la mortalidad entre los mayores, estas cifras podrían elevarse aún más. Por ejemplo, si el lento crecimiento del periodo 2000-2005 se mantuviera hasta que se superaran los 76 años de esperanza de vida, lo que acontecería en 2015, ${ }^{11}$ y a partir de entonces se recuperara el ritmo de crecimiento de la década de los ochenta, en ese caso, la expectativa de vida masculina alcanzaría 86.9 años y la femenina 90.4 en 2050, situándose esta última a tan sólo 2.7 años del máximo mundial. No obstante, también es posible que México nunca se aproxime tanto al liderazgo. A semejanza de Estados Unidos, la excesiva desigualdad socioeconómica que existe en el país (Székely, 1999) podría ocasionar que sólo una parte de la población tuviera acceso a los recursos y a las técnicas sanitarias más avanzadas, y que entonces la esperanza de vida no culminara con una convergencia plena. Desde 1960 la serie femenina de Francia evoluciona a una distancia de entre 1 y 2 años del máximo mundial, igual que la de España desde 1978 y la de Italia desde 1981. Japón incluso se ha situado como líder tras completar su convergencia en

\footnotetext{
${ }^{10}$ Semejantes a las cifras presentadas por Conapo son las del Centro Latinoamericano y Caribeño de Demografía (Celade), que proyecta una esperanza de vida en México de 78.9 años entre la población masculina y de 83.5 entre la femenina para el periodo 2045-2050 (Celade, 2007).

${ }^{11}$ En la proyección de la mortalidad elaborada por Partida (2008: 73) se supone que antes de 2015 sólo mejoran las tasas específicas de mortalidad de los menores de 65 años. De ese modo, la esperanza de vida en 2015 también alcanza los 76 años. Después de 2015 se reanuda el descenso de la mortalidad de los adultos mayores.
} 
1982. Frente a estos ejemplos, la serie femenina de esperanza de vida de Estados Unidos se encuentra en la actualidad a más de 5 años del máximo. En México podría ocurrir algo parecido de mantenerse o agudizarse las diferencias internas en la distribución del ingreso y en el acceso a los medios de salud, lográndose entonces una convergencia incompleta con un posterior avance en paralelo a la serie de máximos, pero muy lejos de ella. ${ }^{12}$

En el futuro, el nivel que alcance la esperanza de vida nacional dependerá de la capacidad propia para innovar y para adoptar y difundir las prácticas más saludables y las técnicas desarrolladas en otros países, pero también de la capacidad de esos países para seguir progresando al ritmo que obliga la tendencia descrita a lo largo de la historia. Alterar el progreso internacional desde México se antoja difícil. Lo que sí puede modificarse es el ritmo de crecimiento de la esperanza de vida nacional, que lleva una década, la femenina, y un lustro, la masculina, creciendo a un ritmo inferior al de la serie de máximos, algo que no ocurría desde el decenio previo a 1920.

Atendiendo a la argumentación anterior, para intentar abandonar la actual situación de desaceleración y retomar la senda de la convergencia con los líderes mundiales en longevidad, habría que adoptar medidas encaminadas a reducir la mortalidad en la vejez, que ha permanecido prácticamente invariada en los últimos años como bien indica Partida (2008: 73). El desarrollo y puesta en funcionamiento de políticas públicas que garanticen la suficiencia financiera de los adultos mayores y su acceso a los medios de salud, ${ }^{13}$ así como la reorientación de los servicios sanitarios hacia el tratamiento de las enfermedades

${ }^{12}$ Llegado este punto cabría cuestionar si esa convergencia incompleta se habrá producido ya. Esto es, si la desaceleración y la leve divergencia observadas durante el último lustro no estarían mostrando el agotamiento de la esperanza de vida nacional para crecer más de prisa que la serie de máximos, dada la elevada desigualdad existente. A pesar de esa opción, dado que la distancia respecto a las poblaciones más avanzadas todavía es muy amplia, y que, por ejemplo, la esperanza de vida en Estados Unidos aún supera en 3 años a la nacional, lo más probable es que la desaceleración advertida se deba, como se ha argumentado, al paso desde la tercera a la cuarta etapa de la transición epidemiológica y no a una culminación incompleta del proceso de convergencia.

${ }^{13}$ En 2006 México era el cuarto país de la Organización para la Cooperación y el Desarrollo Económico (OCDE) que menor porcentaje de su producto interno bruto (PIB) destinaba a gasto en salud: tan sólo 6.6\%; una cifra que únicamente superaba a las de Corea $(6.4 \%)$, Polonia $(6.2 \%)$ y Turquía $(5.7 \%)$, y que se situaba muy lejos de las de Estados Unidos (15.3\%), Suiza (11.3\%) o Francia (11.1\%). Además, era el país en el que menor parte de ese gasto correspondía al sector público: sólo $44.2 \%$ del gasto total destinado a salud (OECD, 2008: 8-9). Combinando ambos indicadores, México resultaba ser el país de la OCDE con menor gasto público en salud en relación con su PIB. 
degenerativas, como la diabetes o las pulmonares obstructivas crónicas, de evolución especialmente adversa en los últimos años, ${ }^{14}$ ayudarían a recuperar el crecimiento de la esperanza de vida y la convergencia con la serie de máximos.

No obstante, y aunque estas acciones serán las que realmente impulsarán el crecimiento de la esperanza de vida a largo plazo, en México se da una particularidad respecto a otras poblaciones que conviene tener en cuenta. Los 74.6 años de expectativa de vida observados en 2005 se alcanzaron con una tasa de mortalidad infantil de $16.8 \%$ según datos del Conapo, una cifra muy elevada si se compara con la de otros países. Como se observa en el cuadro 2, todas las poblaciones analizadas en la Human Mortality Database que han alcanzado en algún momento la actual esperanza de vida de México lo han hecho con tasas de mortalidad infantil sensiblemente inferiores. Sólo España, con una mortalidad infantil de $15.24 \%$ o al alcanzar los 74.58 años de esperanza de vida en 1978, se acerca a las cifras de México en la actualidad. Los demás países se sitúan más lejos, con tasas incluso inferiores a la mitad de la mexicana, como en los casos de Alemania Oriental, Dinamarca, Eslovenia o Finlandia, por ejemplo.

En principio podría pensarse que la diferencia advertida se debe a la comparación de México con poblaciones que se encuentran en fases de desarrollo económico diferentes o que tienen otras culturas o pertenecen a áreas geográficas distantes. Sin embargo, si se utilizan los datos del Centro Latinoamericano y Caribeño de Demografía (Celade), se comprueba que incluso dentro de América Latina la situación de México es excepcional. Ningún país, salvo Panamá en el periodo 2000-2005, ha presentado unas tasas de mortalidad infantil tan altas como las de México al alcanzar los 74 o los 75 años de esperanza de vida (cuadro 3). En algunos casos, como los de Argentina (2000-2005), Barbados (2000-2005), Chile (1990-1995), Martinica (1985-1990) o Puerto Rico (1990-2000), las tasas de mortalidad infantil han resultado ser la mitad de las mexicanas para la misma longevidad de la población. Sólo en Ecuador, Guadalupe y Panamá, además de México, se han superado las veinte defunciones en el primer año de vida por cada mil

${ }^{14}$ La diabetes mellitus, que en 1990 causaba $8.7 \%$ de las muertes registradas entre los mayores de 64 años, en 2006 provoca $15.7 \%$ de los fallecimientos a esas edades. De modo similar, la muerte por enfermedades pulmonares obstructivas crónicas ha elevado su participación en el total de defunciones desde 2.7\% de 1990 hasta 5\% en 2006 (INEGI, 2009). Como comenta Partida (2008: 73), la evolución de estas dos enfermedades en los años más recientes ha resultado tan adversa que ha contribuido de forma negativa a la expansión de la esperanza de vida. 


\section{CUADRO 2}

Tasas de mortalidad infantil en diversos países alrededor de los 74.60 años de esperanza de vida

\begin{tabular}{|c|c|c|c|}
\hline País & Año & Esperanza de vida & $\begin{array}{l}\text { Tasa de mortalidad } \\
\text { infantil (\%o) }\end{array}$ \\
\hline México & 2005 & 74.60 & 16.80 \\
\hline \multirow[t]{2}{*}{ Alemania Occidental } & 1983 & 74.25 & 10.10 \\
\hline & 1984 & 74.82 & 9.62 \\
\hline \multirow[t]{2}{*}{ Alemania Oriental } & 1993 & 74.29 & 6.09 \\
\hline & 1994 & 74.62 & 6.14 \\
\hline \multirow[t]{2}{*}{ Australia } & 1979 & 74.44 & 11.37 \\
\hline & 1980 & 74.60 & 10.76 \\
\hline \multirow[t]{2}{*}{ Austria } & 1986 & 74.49 & 10.27 \\
\hline & 1987 & 74.94 & 9.82 \\
\hline \multirow[t]{2}{*}{ Bélgica } & 1985 & 74.54 & 9.80 \\
\hline & 1986 & 74.74 & 9.77 \\
\hline \multirow[t]{2}{*}{ Canadá } & 1978 & 74.54 & 12.12 \\
\hline & 1979 & 74.90 & 11.17 \\
\hline \multirow[t]{2}{*}{ Dinamarca } & 1981 & 74.29 & 7.60 \\
\hline & 1982 & 74.63 & 8.21 \\
\hline \multirow[t]{2}{*}{ Eslovenia } & 1994 & 73.96 & 6.48 \\
\hline & 1995 & 74.67 & 5.49 \\
\hline \multirow[t]{2}{*}{ España } & 1978 & 74.58 & 15.24 \\
\hline & 1979 & 75.12 & 14.05 \\
\hline \multirow[t]{2}{*}{ Estados Unidos } & 1981 & 74.36 & 12.01 \\
\hline & 1982 & 74.65 & 11.66 \\
\hline \multirow[t]{2}{*}{ Finlandia } & 1983 & 74.46 & 6.16 \\
\hline & 1984 & 74.77 & 6.42 \\
\hline \multirow[t]{2}{*}{ Francia } & 1981 & 74.51 & 10.02 \\
\hline & 1982 & 74.89 & 9.65 \\
\hline \multirow[t]{2}{*}{ Inglaterra y Gales } & 1982 & 74.41 & 10.84 \\
\hline & 1983 & 74.64 & 10.25 \\
\hline \multirow[t]{2}{*}{ Islandia } & 1974 & 74.37 & 11.46 \\
\hline & 1975 & 75.35 & 13.05 \\
\hline \multirow[t]{2}{*}{ Italia } & 1981 & 74.51 & 14.04 \\
\hline & 1982 & 74.98 & 13.13 \\
\hline \multirow[t]{2}{*}{ Japón } & 1975 & 74.41 & 9.80 \\
\hline & 1976 & 74.81 & 9.21 \\
\hline
\end{tabular}


CUADRO 2

(conclusión)

\begin{tabular}{lccc}
\hline País & Año & Esperanza de vida & $\begin{array}{c}\text { Tasa de mortalidad } \\
\text { infantil (\%o) }\end{array}$ \\
\hline Luxemburgo & 1985 & 73.68 & 8.90 \\
Noruega & 1986 & 74.61 & 8.07 \\
& 1973 & 74.43 & 11.16 \\
Nueva Zelanda & 1974 & 74.75 & 9.95 \\
Países Bajos & 1983 & 74.32 & 11.67 \\
Polonia & 1984 & 74.86 & 10.70 \\
& 1975 & 74.52 & 10.11 \\
Portugal & 1976 & 74.65 & 10.40 \\
\multirow{3}{*}{ República Checa } & 2001 & 74.33 & 7.65 \\
\multirow{2}{*}{ Suecia } & 2002 & 74.67 & 7.44 \\
Suiza & 1988 & 74.07 & 13.27 \\
Taiwan & 1989 & 74.63 & 12.28 \\
& 1997 & 74.01 & 5.89 \\
& 1998 & 74.63 & 5.24 \\
& 1969 & 74.15 & 11.50 \\
& 1970 & 74.70 & 11.25 \\
& 1974 & 74.43 & 12.66 \\
& 1975 & 74.82 & 5.08 \\
& 1993 & 74.55 & 5.26 \\
\hline
\end{tabular}

FueNTE: Los datos de México proceden de Partida (2006: 22). Para el resto de las poblaciones se ha recurrido a HMD (2008).

nacimientos una vez alcanzados los 74 años de supervivencia media. No parece, por tanto, que la evolución atípica de la mortalidad infantil responda a un patrón de comportamiento regional, sino a ciertas causas internas.

A partir de la información publicada por el Conapo en la base de datos "Tasa de mortalidad infantil por municipio, 2005", que utiliza el Programa de las Naciones Unidas para el Desarrollo en la elaboración del Índice de Desarrollo Humano a nivel municipal, se puede comprobar que en México conviven poblaciones tan dispares como las de Benito Juárez (Distrito Federal), con una tasa de mortalidad infantil de $3.02 \%$, semejante a la de los países demográficamente más avanzados, y Batopilas (Chihuahua) o Mezquitic (Jalisco), donde la mortalidad de los menores de un año alcanzó 78.83 y $76.66 \%$ respectiva- 


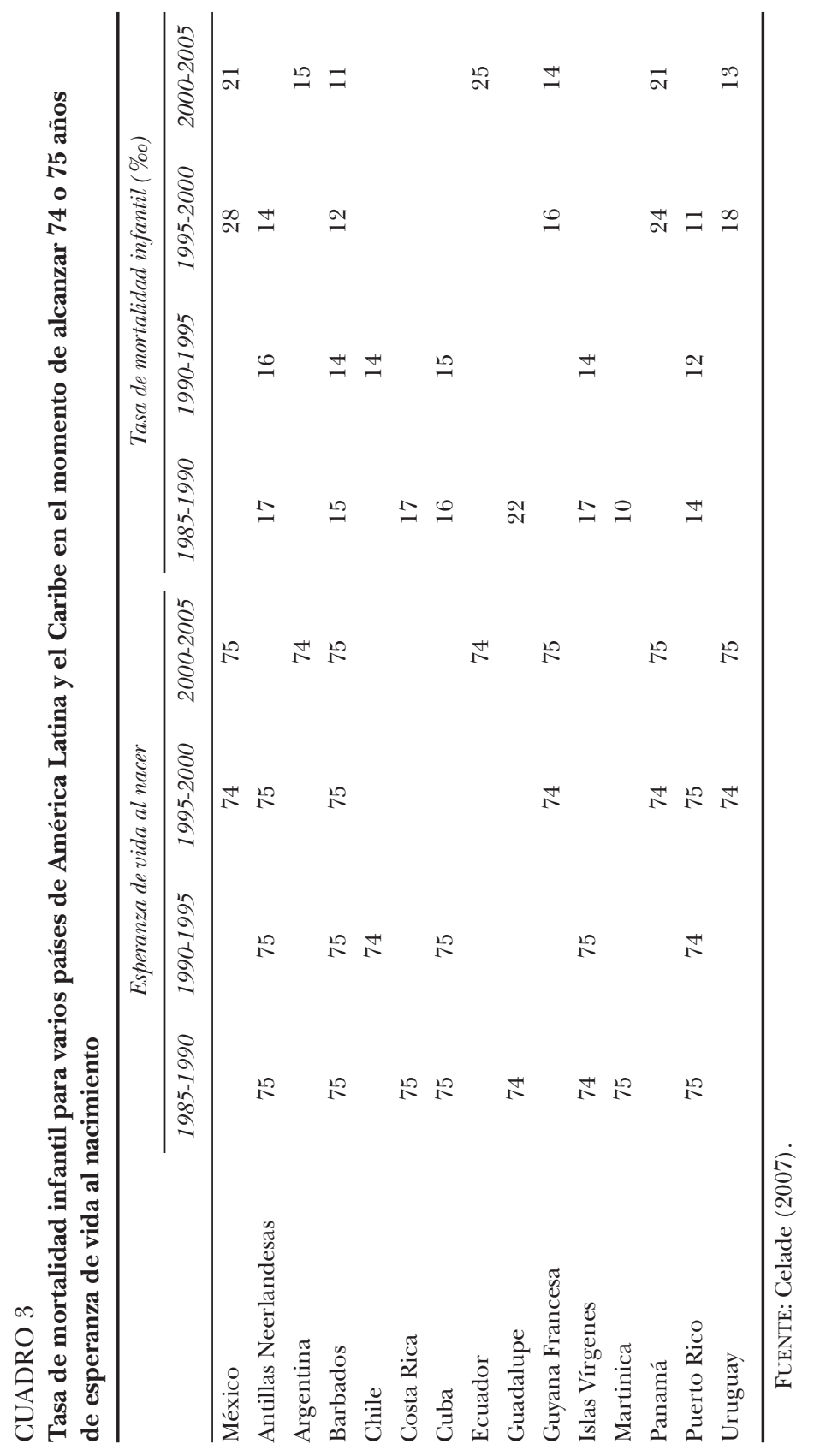


mente, al mismo nivel que Mauritania o Senegal (PNUD, 2007: 265). La Belindia a la que se refiere Bacha (1986) ${ }^{15}$ reproducida en la mortalidad infantil mexicana: con 1529 municipios de los 2454 analizados que se encuentran por encima de las 20 defunciones de entre cada mil nuevos nacimientos; o con entidades federativas como Oaxaca, Chiapas o Guerrero, donde la tasa de mortalidad infantil aún supera $20 \%$ (INEGI, 2007: 92).

Para recuperar el ritmo de crecimiento de la esperanza de vida, y que éste se mantenga a largo plazo, será necesario adoptar medidas que contribuyan a reducir la mortalidad en la vejez, pero sin olvidar que la mortalidad infantil es superior a lo que debería ser en la actual etapa del proceso de modernización demográfica. Por ello es preciso no descuidar la atención a la infancia, especialmente en las regiones más desfavorecidas donde, por ejemplo, todavía existen problemas de desnutrición (Ávila-Curiel et al., 1998; Hernández et al., 2003; Ceipid, 2008). La reducción de la mortalidad entre los menores ayudará en escasa medida a la expansión de la esperanza de vida a largo plazo, puesto que a pesar de lo comentado ya se encuentra en niveles que dificultan la consecución de grandes mejoras. De hecho, dada la actual distribución por edades de las defunciones, aunque la mortalidad durante los cinco primeros años de vida desapareciera por completo, ceteris paribus, la expectativa de supervivencia no se elevaría en más de dos años. No obstante, al tiempo que se afronte en el futuro de forma decidida la reorientación de los servicios de salud hacia el tratamiento de las enfermedades crónicas y degenerativas, deberán adoptarse medidas tendentes a combatir la mortalidad infantil, más elevada que en el resto de las poblaciones.

\section{Conclusiones}

En este artículo se ha analizado la evolución de la esperanza de vida al nacimiento en México a partir de la relación que la serie nacional mantiene con las de otros países más avanzados en la lucha contra la mortalidad. Se ha explicado que el rápido aumento de la expectativa de vida que tuvo lugar a mediados del pasado siglo fue posible por el

${ }^{15}$ Bacha (1986) rebautizó a Brasil con el nombre de Belindia en un intento por reflejar la realidad socioeconómica dual que caracterizaba a su país, donde unos pocos disfrutaban de unos ingresos semejantes a los de un belga promedio, mientras la mayoría apenas subsistía con los niveles de renta propios de la India. 
retraso acumulado respecto a las poblaciones líderes, y que la asimilación de costumbres más saludables y el acceso a las técnicas desarrolladas durante años en otras naciones hizo posible que se recortara la distancia existente, aunque se fue reduciendo el margen de mejora y, en consecuencia, el ritmo de crecimiento de la serie nacional en años posteriores.

Para conocer la capacidad de aumento que en el futuro tendrá la esperanza de vida en México se ha analizado la serie de máximos mundiales de 1840 a 2006, y se ha encontrado que la misma puede modelizarse mediante una función logística de dos asíntotas que se ajusta a las observaciones mejor que el esquema lineal propuesto por Oeppen y Vaupel (2002). Tomando la esperanza de vida máxima mundial como referencia de la capacidad de progreso de la serie nacional y atendiendo a la experiencia de otros países, se ha concluido que la divergencia observada en la década más reciente respecto a la serie de máximos responde al tránsito de la tercera a la cuarta etapa de la transición epidemiológica, y que lo más probable es que en el futuro, al igual que en otras poblaciones, se supere esta fase de lento crecimiento y se reanude la convergencia. No obstante, para que ello ocurra será necesario garantizar la suficiencia financiera y el acceso de los adultos mayores a los medios de salud, así como favorecer la reasignación de recursos desde el diagnóstico y el tratamiento de las enfermedades infecciosas y parasitarias hasta las crónicas y degenerativas, de importancia creciente entre las causas de fallecimiento. Finalmente, se ha advertido que, pese a la necesidad de impulsar el aumento de la esperanza de vida reduciendo la mortalidad en la vejez, no conviene descuidar la atención a los más jóvenes, ya que la mortalidad infantil presenta un valor muy superior al que cabría esperar del actual nivel de esperanza de vida al nacimiento, dada la experiencia de otras poblaciones.

\section{Bibliografía}

Arriaga, Eduardo E. (1968), New Life Tables for Latin American Populations in the Nineteenth and Twentieth Centuries, Berkeley, University of California Press.

Arriaga, Eduardo E. y Kingsley Davis (1969), "The Pattern of Mortality Change in Latin America”, Demography, vol. 6, núm. 3, pp. 223-242.

Ávila Curiel, Abelardo, Teresa Shamah Levy, Carlos Galindo Gómez, Gerardo Rodríguez Hernández y Linda M. Barragán Heredia (1998), "La desnu- 
trición infantil en el medio rural mexicano", Salud Pública de México, vol. 40, núm. 2, pp. 150-160.

Bacha, Edmar L. (1986), "El economista y el rey de Belindia: una fábula para tecnócratas”, en Edmar L. Bacha (coord.), El milagro y la crisis: economía brasileña y latinoamericana, México, Fondo de Cultura Económica, cap. 1, pp. 13-17.

Bagnall, Roger S. (1993), Egypt in Late Antiquity, Princeton, Princeton University Press.

Benítez, Raúl y Gustavo Cabrera (1967), Tablas abreviadas de mortalidad de la población de México, 1930, 1940, 1950, 1960, México, El Colegio de México.

Bourgeois-Pichat, Jean (1952), "Essai sur la mortalité 'biologique' de l'homme", Population, vol. 7, núm. 3, pp. 381-394.

Bulatao, Rodolfo A., Eduard Bos, Patience W. Stephens y My T. Vu (1989), "Projecting Mortality for all Countries", Policy, Planning, and Research Working Papers, núm. 337, Washington, World Bank.

Camposortega, Sergio (1992), Análisis demográfico de la mortalidad en México 1940-1980, México, El Colegio de México.

Ceipid (2008), Informe de desnutrición en los municipios más vulnerables de Chiapas, San Cristóbal de Las Casas, Centro de Investigación sobre Pobreza y Desarrollo.

Celade (2007), Información demográfica: estimaciones y proyecciones, Santiago de Chile, Centro Latinoamericano y Caribeño de Demografía, División de Población <www.eclac.cl/celade/proyecciones/basedatos_BD.htm>.

Conapo (2009), México en cifras: indicadores demográficos básicos, México, Consejo Nacional de Población <www.conapo.gob.mx $>$.

Corona, Rodolfo, René Jiménez y Alberto Minujín (1982), La mortalidad en México, México, Instituto de Investigaciones Sociales, UNAM.

Galor, Oded y David N. Weil (1999), "From Malthusian Stagnation to Modern Growth”, American Economic Review, vol. 89, núm. 2, pp. 150-154.

Galor, Oded y David N. Weil (2000), "Population, Technology, and Growth: From Malthusian Stagnation to the Demographic Transition and Beyond", American Economic Review, vol. 90, núm. 4, pp. 806-828.

Gerland, Patrick (2005), "From Divergence to Convergence in Sex Differentials in Adult Mortality in Developed Countries", trabajo presentado en la XXV Conferencia Internacional de Población, Tours, 18 a 23 de julio.

Hernández, Daniel, Cristina Barberena, José Ángel Camacho y Hadid Vera (2003), "Desnutrición infantil y pobreza en México", Cuadernos de Desarrollo Humano, núm. 12.

HMD (2008), Human Mortality Database, Berkeley-Rostock, University of California/Max Planck Institute for Demographic Research. Disponible en $<$ www.mortality.org $>$ y en <www.humanmortality.de $>$.

INEGI (2007), Mujeres y hombres en México 2007, México, Instituto Nacional de Estadística, Geografía e Informática. 
INEGI (2009), Estadísticas de mortalidad, México, Instituto Nacional de Estadística, Geografía e Informática <www.inegi.org.mx>.

Kerbo, Harold R. (2003), Estratificación social y desigualdad. El conflicto de clase en perspectiva histórica, comparada y global, Madrid, McGraw Hill.

Lee, Ronald D. (2003), "Mortality Forecasts and Linear Life Expectancy Trends", Documento de Trabajo, núm. 2003-0003CL, Berkeley, University of California, Center for the Economics and Demography of Aging.

Livi-Bacci, Massimo (2002), Historia mínima de la población mundial, Barcelona, Ariel.

Malthus, Thomas R. (1970), Primer ensayo sobre la población, Madrid, Alianza.

Meslé, France (2004), "Espérance de vie: un avantage féminin menacée”, Population et Sociétés, núm. 402.

Morelos, José B. (1994), "La mortalidad en México: hechos y consensos", en Francisco Alba y Gustavo Cabrera (comps.), La población en el desarrollo contemporáneo de México, México, El Colegio de México, pp. 53-84.

Notestein, Frank W. (1945), "Population: The Long View", en Theodore W. Schultz (ed.), Food for the World, Chicago, University of Chicago Press, pp. 36-57.

OECD (2008), OECD in Figures 2008, París, OECD Publications.

Oeppen, Jim y James W. Vaupel (2002), "Broken Limits to Life Expectancy", Science, vol. 296, núm. 5570, pp. 1029-1031.

Oliver, Lilia (1988), "La mortalidad en Guadalajara, 1800-1850", en Mario Bronfman y José Gómez de León (comps.), La mortalidad en México: niveles, tendencias y determinantes, México, El Colegio de México, pp. 167-202.

Olshansky, S. Jay y A. Brian Ault (1986), "The Fourth Stage of Epidemiologic Transition: The Age of Delayed Degenerative Diseases", The Milbank Memorial Fund Quarterly, vol. 64, núm. 3, pp. 355-391.

Olshansky, S. Jay, Bruce A. Carnes y Christine Cassel (1990), "In Search of Methuselah: Estimating the Upper Limits to Human Longevity", Science, vol. 250, núm. 4981, pp. 634-640.

Olshansky, S. Jay, Bruce A. Carnes y Aline Désesquelles (2001), "Prospects for Human Longevity", Science, vol. 291, núm. 5508, pp. 1491-1492.

Omran, Abdel R. (1971), "The Epidemiologic Transition: A Theory of the Epidemiology of Population Change”, The Milbank MemorialFund Quarterly, vol. 49, núm. 4, pp. 509-538 [reimpreso en The Milbank Quarterly (2005), vol. 83, núm. 4, pp. 731-757].

Ortes, Giammaria (1966), Riflessioni sulla populazione delle nazioni per rapporto all'economia nazionale [1790], Roma, Ateneo e Bizzarri.

Partida, Virgilio (2006), Proyecciones de la población de México 2005-2050, México, Consejo Nacional de Población.

Partida, Virgilio (2008), Proyecciones de la población de México, de las entidades federativas, de los municipios y de las localidades, 2005-2050 (documento metodológico), México, Consejo Nacional de Población.

PNUD (2007), Informe sobre desarrollo humano 2007-2008, Nueva York, PNUD. 
SCOR (2004), "Mortality Forecasts and Life Annuities", Technical Newsletters, núm. 19 (Life Collection).

Spengler, Joseph J. (1978), "Los factores demográficos y el primer desarrollo económico moderno", en David V. Glass y Roger Revelle (dirs.), Población y cambio social: estudios de demografía histórica, Madrid, Tecnos, pp. 96-107.

Spiegel, Henry W. (1973), El desarrollo del pensamiento económico, Barcelona, Omega.

Székely, Miguel (1999), "La desigualdad en México: una perspectiva internacional", Documento de Trabajo, Washington, Banco Interamericano de Desarrollo.

Vallin, Jacques (1995), La demografía, Madrid, Alianza.

White, Kevin M. (2002), "Longevity Advances in High-Income Countries, 195596”, Population and Development Review, vol. 28, núm. 1, pp. 59-76.

Wilson, Chris (2001), "On the Scale of Global Demographic Convergence 19502000", Population and Development Review, vol. 27, núm. 1, pp. 155-171.

Zamora, Francisco (1994), "Proyecciones demográficas", en Julio Vinuesa (ed.), Demografia: análisis y proyecciones, Madrid, Síntesis, pp. 237-358. 